\title{
Mesoporous silica coatings for controlled release of the antibiotic ciprofloxacin from implants
}

\author{
Nina Ehlert, $\dagger^{a}$ Muhammad Badar, $\dagger^{b}$ Anne Christel, ${ }^{a}$ Sven Jare Lohmeier, ${ }^{a}$ Tammo Luessenhop, ${ }^{a}$ Martin Stieve, ${ }^{c}$ \\ Thomas Lenarz, ${ }^{c}$ Peter Paul Mueller ${ }^{b}$ and Peter Behrens $* a$
}

\author{
Received 17th May 2010, Accepted 27th September 2010 \\ DOI: 10.1039/c0jm01487g
}

To generate bioactive coatings for medical implants, a novel procedure has been developed using a coating of mesoporous silica for controlled drug delivery. Plain glass slides were used as substrates. The mesoporous coatings were then loaded with the antibacterial drug ciprofloxacin. The drug release kinetics were investigated in a physiological buffered solution. The drug loading capacity of the unmodified mesoporous coatings was low but could be increased nearly ten-fold (to about $2 \mu \mathrm{g} \mathrm{cm}^{-2}$ of the macroscopic surface) by functionalizing the mesoporous surface with sulfonic acid groups. To achieve a controlled drug release over an extended time period, further coatings were added. Covering the surface of the drug loaded mesoporous silica layer by dip-coating with bis(trimethoxysilyl)hexane resulted in an organosiloxane layer which retarded the release for up to 30 days. By an additional evaporation coating with dioctyltetramethyldisilazane, the release of ciprofloxacin was prolonged for up to 60 days. The biocompatibility of the different coatings was tested in cell culture assays. The presence of the additional silane-derived hydrophobic coatings somewhat reduced the biocompatibility. The antibacterial efficacy of the materials was demonstrated by using clinically relevant biofilm-forming pathogenic bacteria. A test where the sequential release of ciprofloxacin (in 2 days intervals) and the bacterial viability were tested in parallel showed good concordance in the results. The material where a sulfonate-functionalized mesoporous silica layer is loaded with ciprofloxacin and then coated by an organosiloxane layer derived from bis(trimethoxysilyl)hexane showed the best results with regard to antibacterial efficacy and will further be tested in animal experiments.

\section{Introduction}

Microbial biofilms on clinical implants pose a major clinical problem which is difficult to treat. ${ }^{\mathbf{1 , 2}}$ Numerous strategies have been devised to prevent biofilm formation by coating the implant surfaces with antimicrobial substances. Mesoporous silica materials offer a novel opportunity for this purpose. They are currently being investigated as controlled drug release systems due to their unique properties as high surface area, tunable pore size with narrow distribution in the nanometre range and adaptable surface chemistry based on functionalization of the silanol groups present on the silica surface. Mesoporous silica shows a good biocompatibility in animal experiments. ${ }^{3-5}$ Usually, mesoporous silicas are studied in the form delivered by solution-phase syntheses, namely as powders, or, in some cases, as mesoporous hollow silica spheres. ${ }^{6-8}$ These material forms are, however, difficult to apply in combination with a pre-formed implant or prosthesis. For this purpose, mesoporous silica films, deposited on the surface of the implant, present a promising

anstitut für Anorganische Chemie, Leibniz Universität Hannover, Callinstraße 9, 30167 Hannover, Germany.E-mail: peter.behrens@acb. uni-hannover.de; Fax: +49511 7623006; Tel: +49511 7623697

${ }^{b}$ Helmholtz-Zentrum für Infektionsforschung, Inhoffenstraße 7, 38124 Braunschweig, Germany

${ }^{c}$ Hals-Nasen-Ohren-Klinik, Medizinische Hochschule Hannover, CarlNeuberg-Straße 1, 30625 Hannover, Germany

$\uparrow$ These two authors contributed equally to this work. practical approach. ${ }^{3-5}$ For example, ossicular replacement prostheses often have to be implanted into an infected middle ear. These often chronic infections play an important part in the implantation of alloplastic materials used for ossicular replacement. ${ }^{9,10}$ Therefore, a systemic antibiotics therapy, which is stressful for the body, is typically applied after the implantation. In the ear region, chronic infections are caused primarily by the difficult pathogen Pseudomonas aeruginosa, which is treatable with the antibiotic ciprofloxacin. Local delivery of an antibiotic from the freshly implanted prosthesis will help in combating this problematic bacterial flora and thus assist in the healing process.

The loading and the release of drugs from mesoporous silica can be controlled in different ways, as shown in many investigations. In addition to the size and the shape of the pores and particles, ${ }^{11-13}$ the chemical interaction of the materials surface with the drug is the most important factor. ${ }^{14}$ Silica surfaces can be equipped with functional residues by grafting, i.e. the postsynthetic reaction of functionalized silanes with the silanol groups on the surface, or by co-condensation in a one-pot synthesis; both methods are currently being discussed in view of their advantages and disadvantages. ${ }^{14-17}$ The different modifications can be used to optimize the amount of drug taken up and delivered by mesoporous silica-based drug delivery systems. Furthermore, they can influence the kinetics of drug release. A variety of modifications is possible. For example, a hydrophobic character can be imparted to the silica surface by the attachment of unreactive groups (e.g. trimethylsily $\left.{ }^{18-20}\right)$. In addition, 
1.

2. $\mathrm{H}_{2} \mathrm{O}_{2}$
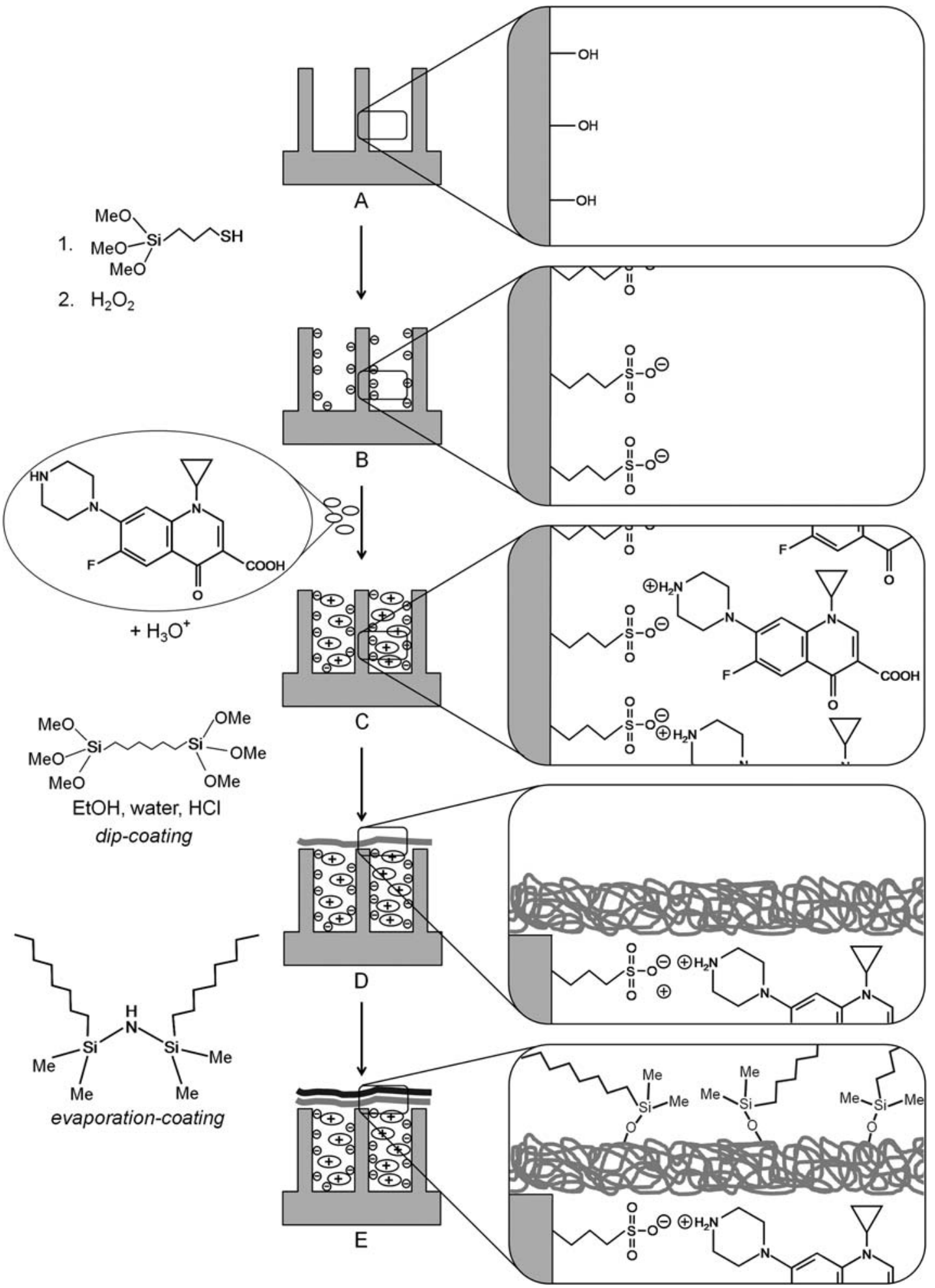

Fig. 1 Scheme showing the different materials used in this study. Different modifications were carried out to achieve a high loading and a controlled release of ciprofloxacin from mesoporous silica films. Glass substrates were functionalized successively with a mesoporous silica film (sample A), by the introduction of sulfonic acid groups (sample B), by loading with ciprofloxacin (sample C), by the application of a surface layer derived from bis(trimethoxysilyl)hexane by dip-coating (sample D), and by the additional application of a surface layer derived from dioctyltetramethyldisilazane by evaporation coating (sample E).

negatively charged groups (like carboxyl ${ }^{21,22}$ ), positively charged ones (as protonated amino groups ${ }^{16,17,23}$ ) or reactive groups (as epoxy $^{24}$ or thiol residues ${ }^{25}$ ) can be used to tailor the surface properties in order to match the properties of the drug to be delivered. The density of these functionalities has to be adjusted carefully. Especially in the attachment of charged groups, the net 
charge of the surface is a crucial factor, where also the unreacted silanol groups retain their influence. ${ }^{26}$ For example, the loading of a drug consisting of negatively charged molecules can be enhanced when positive charges are placed on the surface, ${ }^{23,27,28}$ drug molecules with extended hydrophobic parts can be attracted to the surface by the presence of hydrophobic residues. ${ }^{\mathbf{1 8 2 9}}$ The release of a drug to an aqueous medium can be retarded by a protective outer hydrophobic layer which hinders the surrounding aqueous media from entering the pores. ${ }^{20,30}$

Here we report a practical approach for equipping implants with the ability to fight bacterial infections by local delivery of ciprofloxacin, a broad spectrum antibiotic, from a specially developed mesoporous silica coating (see scheme in Fig. 1). Such silica coatings can be applied to standard implant materials, e.g. Bioverit ${ }^{\circ}$ II, a mica-containing glass ceramic from which ossicular replacement prostheses and other implants are routinely fabricated. ${ }^{31,32}$ We use a sulfonic acid modification to increase the amount of loaded ciprofloxacin in a mesoporous silica layer and show its biocompatibility and its effectiveness in combating bacteria in vitro. Additional coatings generated from silanes are added to attenuate the typical initial burst release behavior of drug delivery systems and retard the delivery of the drug.

\section{Experimental}

\section{Mesoporous silica layer}

As base material for the coatings glass substrates (Glasbearbeitung Henneberg \& Co., Martinroda, Germany) were used. The 3mercaptotrimethoxysilane, 1,3-di-n-octyltetramethyldisilazane and the bis(trimethoxysilyl)hexane were ordered from ABCR GmbH \& Co. KG (Karlsruhe, Germany). Absolute ethanol was purchased from Merck (Darmstadt, Germany). All other chemicals were obtained from Sigma-Aldrich Chemie GmbH (Munich, Germany). All chemicals were used without further purification.

For the compilation of in vitro release profiles standard glass slides $(76 \mathrm{~mm} \times 26 \mathrm{~mm})$ and for cell culture experiments glass disks $(10 \mathrm{~mm} \times 10 \mathrm{~mm})$ with a height of $0.95 \mathrm{~mm}$ were employed. In a further experiment, where we determined simultaneously the release properties and the antibacterial efficacy, the release profiles were also determined using the glass disks described. The different substrates were first coated with mesoporous silica layers. Prior to the coating, all specimens were cleaned in an ultrasonic bath, first in absolute ethanol and then in acetone for ten minutes each.

The solution used for the preparation of nanostructured silica coatings contained ethanol, water, hydrochloric acid, tetraethoxysilane (TEOS) as a silica source and poly(ethylene glycol)poly(propylene glycol)-block-co-polymer (Sigma-Aldrich, $\mathrm{EO}_{20} \mathrm{PO}_{70} \mathrm{EO}_{20}$, average $M_{\mathrm{n}} \approx 5800$, similar to Pluronic $\AA \mathrm{P}-123$, $\mathrm{BASF})$ as the structure-directing agent. ${ }^{33}$ The dip-coating solution had a molar composition of TEOS : EtOH : $\mathrm{H}_{2} \mathrm{O}$ : $\mathrm{HCl}: \mathrm{EO}_{20} \mathrm{PO}_{70} \mathrm{EO}_{20}=1: 48.9: 26.9: 0.06: 0.0135$. It was prepared by adding TEOS to $\mathrm{EO}_{20} \mathrm{PO}_{70} \mathrm{EO}_{20}$ dissolved in a mixture of ethanol, water and hydrochloric acid; the solution was stirred for about ten minutes before coating the specimens. Glass samples were coated using a dip-coating procedure, employing a DC Small Dip-Coater with $75 \mathrm{~mm}$ travel from NIMA (Coventry, England), operated in a climate box at a constant humidity adjusted by $50 \% \mathrm{w} / \mathrm{w}$ glucose solution. The samples were immersed in the coating solution and then withdrawn perpendicular to the surface of the solution with a speed of approximately $1 \mathrm{~mm} \mathrm{~min}{ }^{-1}$. The samples were then left at constant humidity for five minutes. After coating, the specimens were dried at $60{ }^{\circ} \mathrm{C}$ overnight, followed by calcination at $415^{\circ} \mathrm{C}$ for four hours (rate of heating/cooling $1{ }^{\circ} \mathrm{C} \mathrm{min}^{-1}$ ). This material, consisting of the mesoporous silica layer only, is designated as "A" in Fig. 1 and in the following.

\section{Sulfonic acid modification}

The sulfonic acid modification was carried out according to ref. 34. The following procedure was applied in parallel for five glass slides. The glass slides were cooled to $0{ }^{\circ} \mathrm{C}$ in $45 \mathrm{ml}$ of dichloromethane before $5.9 \mathrm{ml}$ of 3-mercaptotrimethoxysilane were added. The solution was gently stirred for $22 \mathrm{~h}$ without renewing the ice bath. Then the glass slides were washed with dichloromethane and absolute ethanol and dried at $100{ }^{\circ} \mathrm{C}$ for five hours. $50 \mathrm{ml}$ of hydrogen peroxide $(30 \% \mathrm{w} / \mathrm{w})$ were added and allowed to react for $48 \mathrm{~h}$ followed by washing with water and absolute ethanol. Finally the glass slides were dried at $60{ }^{\circ} \mathrm{C}$ for two hours and cooled to room temperature before the insertion of ciprofloxacin. This material, consisting of the sulfonatemodified mesoporous layer, is designated as " $\mathrm{B}$ " in Fig. 1 and in the following.

\section{Drug loading procedure}

The insertion of ciprofloxacin was carried out in a $60 \mathrm{mM}$ solution of the drug at $\mathrm{pH} 4$ and at $37{ }^{\circ} \mathrm{C}$ for three days, either with samples presenting the unmodified mesoporous layer or the sulfonate-modified one. The solution was prepared as follows: $10 \mathrm{~g}$ of ciprofloxacin were added to about $300 \mathrm{ml}$ of water and the $\mathrm{pH}$ value was decreased with hydrochloric acid ( $2 \mathrm{M})$ until a clear yellow solution was formed $(\mathrm{pH} \approx 2)$. Then the $\mathrm{pH}$ was adjusted to 4 with sodium hydroxide solution ( $1 \mathrm{M})$, thereby approaching a volume of $500 \mathrm{ml}$. Finally the solution was supplemented to $500 \mathrm{ml}$.

After the insertion, the glass slides were rinsed with $50 \mathrm{ml}$ of water for each side to wash off the highly concentrated solution at the outer surface of the glass slide. Afterwards the glass slides were dried for two hours at room temperature at constant air humidity adjusted with $50 \% \mathrm{w} / \mathrm{w}$ glucose. Then, these samples were either transferred to the release experiment or modified further as described below. The drug-loaded material based on sulfonate-modified layer is referred to as " $\mathrm{C}$ " in Fig. 1 and in the following.

\section{Controlled release modifications}

To produce a hydrophobic layer on the surface of the mesoporous layer, it was treated with bis(trimethoxysilyl)hexane using a dip-coating procedure, following ref. 30. For this purpose, a solution containing $8.7 \mathrm{ml}$ bis(trimethoxysilyl)hexane, $31.2 \mathrm{ml}$ ethanol, $1.29 \mathrm{ml}$ water and $1.614 \mathrm{ml}$ hydrochloric acid $(0.1 \mathrm{M})$ was stirred for $30 \mathrm{~min}$. Glass slides were then dipped into the solution individually and withdrawn with an approximate speed 
of $2 \mathrm{~cm} \mathrm{~s}^{-1}$ to avoid leaching of the inserted ciprofloxacin. The samples were dried for twelve hours at room temperature at constant air humidity adjusted with $50 \% \mathrm{w} / \mathrm{w}$ glucose. Then, they were either transferred to the release experiment or modified further as described below. This material, consisting of the drugloaded sulfonate-modified mesoporous layer and the hydrophobizing silane-derived layer is referred to as "D" in Fig. 1 and in the following.

For the further modification, samples of type D were treated with dioctyltetramethyldisilazane (1,3-di- $n$-octyltetramethyl-disilazane) via the vapour phase. For this purpose, $6 \mathrm{ml}$ of the silazane were put into a $300 \mathrm{ml}$ Erlenmeyer flask (broad-necked) and heated to $52{ }^{\circ} \mathrm{C}$ for ten hours. During this time, five samples were hanging above the liquid at the upper end of the flask at a distance of about $15 \mathrm{~cm} .{ }^{20}$ After drying for ten minutes at ambient conditions, the samples were transferred to the release experiment. This material, based on material $\mathrm{D}$, but carrying another silazane-derived surface layer is referred to as " $E$ " in Fig. 1 and in the following.

\section{Ciprofloxacin release}

The release measurements were conducted as follows: five glass slides were placed into $45 \mathrm{ml}$ of a pre-warmed solution $\left(37^{\circ} \mathrm{C}\right)$ of $0.01 \mathrm{M}$ phosphate buffered saline (PBS) and kept at this temperature. The measurements of the ciprofloxacin concentration in the solution took place after fixed time intervals of 15,35 , $55,75,135,195$ and $315 \mathrm{~min}$. Afterwards, the measurements were performed every $24 \mathrm{~h}$. The quantitative determination of ciprofloxacin in the solution was carried out on a spectrophotometer UV-mini 1240 (Shimadzu, Duisburg, Germany) at $275 \mathrm{~nm}$. The whole medium was replaced after each measurement to simulate dynamic fluid conditions in the body.

In a further experiment, where we determined simultaneously the release properties and the antibacterial efficacy, the release profiles were determined on $10 \mathrm{~mm} \times 10 \mathrm{~mm}$ glass disks, similar to those which were used in the bacteria culture experiment. The samples were incubated in $0.5 \mathrm{ml} \mathrm{PBS}$ and the medium was replaced every $48 \mathrm{~h}$ for a period of ten days. Blank values determined on ciprofloxacin-free sulfonated mesoporous silica substrates were subtracted.

\section{Biocompatibility testing}

For biocompatibility testing, we studied the behavior of the sulfonated base material (sample B) in comparison with ciprofloxacin loaded materials (samples C, D and E). Samples were transferred to a 24-well plate. The sulfonic acid modified surface and cell culture plastic (CCP) were used as controls. Briefly, a near confluent culture of NIH3T3 cells was detached with trypsin and the resulting suspension was diluted to $1: 5$ in fresh cell culture medium. One millilitre of this cell suspension was added to each of the wells containing the glass disks. The disks were incubated with these cells at $37{ }^{\circ} \mathrm{C}$ in a cell culture incubator. Images of the cells were taken with an Axio Observer.A1 microscope (Carl Zeiss, Oberkochen, Germany) at 24 and $72 \mathrm{~h}$ intervals after inoculation of the cells. The experiment was repeated three times. Additionally, corresponding ciprofloxacinfree samples were tested as a control. Sample D without ciprofloxacin is assigned as F, sample E as G. Sample $\mathrm{H}$ is equipped with the evaporation coating with dioctyltetramethyldisilazane applied on sample E, but without the bis(trimethoxysilyl)hexane coating below.

\section{Antibacterial efficacy}

For testing the efficacy of the different materials against bacteria, glass disks with the mesoporous coating (sample A), with additional sulfonic acid modification (sample B) and with that same material loaded with ciprofloxacin (sample $\mathrm{C}$ ) were placed in a 24-well plate. Plane glass disks were used as control. Each of these disks was incubated with $100 \mu \mathrm{l}$ of a bacterial suspension of $\mathrm{OD}_{600}=0.2$ of P. aeruginosa (PAO1 CTX::1ux) in PBS for 15 $\mathrm{min}$ at room temperature. After $15 \mathrm{~min}, 0.9 \mathrm{ml}$ of LB medium $\left(\right.$ Luria Broth ${ }^{35}$ ) was added into each well and the multi-well plate was placed on a shaker at $60 \mathrm{rpm}$ at $37^{\circ} \mathrm{C}$. The plate was observed under the IVIS-200 (Xenogen Corp., Alameda, Canada) after six hours and the luminescence was measured individually for each well.

For the investigation of the long-term antimicrobial efficacy of the coatings, samples of sulfonic acid-modified mesoporous silica films containing ciprofloxacin (sample C), and corresponding samples modified in addition by dip-coating with bis(trimethoxysilyl)hexane (sample D) and furthermore by evaporation coating with dioctyltetramethyldisilazane (sample E) were used. Disks of each of these materials were placed in $0.5 \mathrm{ml}$ of LB medium and the supernatant was replaced every $48 \mathrm{~h}$ up to ten days. The supernatants collected individually from different samples and after different release periods were then added to $10 \mu \mathrm{l}$ of a suspension of $P$. aeruginosa (PAO1 CTX::lux) at an $\mathrm{OD}_{600}$ of $0.2 .90 \mu \mathrm{l}$ of each supernatant were used. Immediately after the addition and after six hours at $37^{\circ} \mathrm{C}$, the luminescence of each well was determined to determine the bacterial viability which is given relative to the value of ciprofloxacin-free mesoporous silica, which was set to 1.0.

\section{Characterization methods}

The mesostructured layers were investigated by $\mathrm{X}$-ray diffraction (XRD). The samples were measured on a Stoe $\theta-\theta$-diffractometer (Darmstadt, Germany) in reflection geometry. A secondary beam monochromator (graphite) was applied to produce $\mathrm{Cu} \mathrm{K} \alpha$ radiation. Krypton adsorption/desorption measurements were performed at $77 \mathrm{~K}$ with an Autosorb-1-MP instrument (Quantachrome Instruments, Boynton Beach, Florida, USA). The analysis station was equipped with high-precision pressure transducers to ensure a highly accurate determination of the adsorbed amount. During the equilibration time the sample cell was isolated to minimize the effective dead volume. The saturation pressure $p_{0}$ was given as $2.63 \mathrm{~mm} \mathrm{Hg}$ ( $p_{0}$ of supercooled Krypton). Measurements were performed in the relative pressure range from 0.025 to 0.400 . Before the measurement, the samples were outgassed at $200{ }^{\circ} \mathrm{C}$ for 24 hours. The relative pressure range of $p / p_{0}=0.05$ to 0.30 was chosen to determine the specific BET surface area $S_{\mathrm{BET}, \mathrm{Kr}}$. Static contact angle measurements were performed on a Surftens universal contact angle goniometer (OEG, Frankfurt/Oder, Germany) with water as the probing liquid. On every glass slide, the contact angle was measured at 


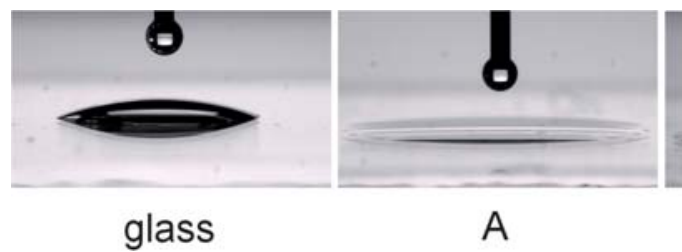

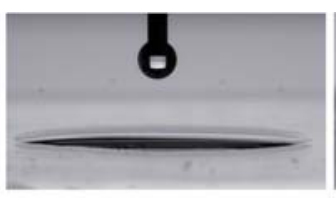

B

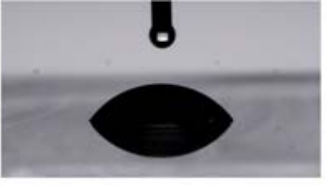

D

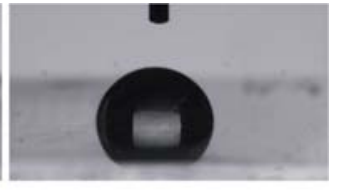

E

Fig. 2 Static contact angle measurements on a cleaned glass substrate, equipped with a mesoporous silica layer (A), after modification with sulfonic acid groups (B), covered with a dip-coated layer derived from bis(trimethoxysilyl)hexane (D), in addition covered with a layer obtained by evaporation coating with dioctyltetramethyldisilazane (E).

five different positions, all at least five millimetres away from the edges of the glass slide, and a representative image was chosen. The thickness of the mesoporous silica film was measured with a stylus profiler DETAK6M from Veeco instruments Inc. (Plainview, USA) with a force of $9 \mathrm{mg}$, duration of 100 seconds and a length of $3000 \mu \mathrm{m}$ per measurement. The quantification of sulfonic acid residues was carried out by methylene blue adsorption according to ref. 36 . Unmodified and sulfonic acidmodified mesoporous silica layers on glass substrates $(26 \times 10$ $\mathrm{mm}$ ) were each placed into $1.5 \mathrm{ml}$ of a $0.03 \mathrm{mM}$ solution of methylene blue in $0.01 \mathrm{M}$ hydrochloric acid. The amount of adsorbed dye was determined by the difference of methylene blue concentration after $12 \mathrm{~h}$. The calculated value is a mean of nine samples. The blank values determined on unmodified mesoporous silica layers were subtracted.

\section{Results and discussion}

\section{Characterization of the mesoporous silica layer}

$\mathrm{X}$-Ray diffraction measurements gave results similar to those as described in detail elsewhere, ${ }^{37}$ thus confirming the presence of the mesostructure. Further investigations with a profilometer revealed that a silica film as produced by our method on a glass slide has a layer thickness varying between 30 and $150 \mathrm{~nm}$. Sorption measurements revealed that the film has an inner surface of $11.2 \mathrm{~cm}^{-2}$ per $\mathrm{cm}^{-2}$ of the substrate, which shows a large increase of the surface area. A typical amount of silanol groups of calcined mesoporous materials is two to four silanol groups per $\mathrm{nm}^{-2}$. This leads to a value of 2.2 to $4.5 \times 10^{15}$ silanol groups per $\mathrm{cm}^{-2}$ of the substrate. The experimental quantification of the number of sulfonic acid residues was carried out by methylene blue adsorption. ${ }^{36}$ This assay gives a value of approx. $2.4 \times 10^{15}$ sulfonic acid groups per $\mathrm{cm}^{-2}$ of the substrate. Thus, more than half of the silanol groups present were transferred into sulfonic acid groups.

The different surface modifications carried out during the whole procedure were monitored by static contact angle measurements. The results show drastic changes in surface properties (Fig. 2). Initially, the hydrophilic surface of the mesoporous silica layer can be discerned from the decrease of the contact angle from about $20^{\circ}$ (Fig. 2, glass) for the cleaned glass substrate to less than $5^{\circ}$ for the mesoporous silica layer (Fig. 2, sample A). After the first modification steps producing sulfonic acid groups, the surface stays hydrophilic with only a slight increase of the contact angle which remains below $5^{\circ}$ (Fig. 2, sample B). Although propyl groups are also introduced onto the surface, the hydrophilic sulfonic acid groups seem to dominate the surface chemistry. After dip-coating with bis(trimethoxysilyl)hexane, the surface exhibits hydrophobic properties with contact angles between $50^{\circ}$ and $55^{\circ}$ (Fig. 2, sample D). The contact angle increases further after the evaporation coating with dioctyltetramethyldisilazane in the last step (Fig. 2, sample E), yielding values of more than $90^{\circ}$.

\section{Controlled release profiles}

Spectrophotometric analyses of the PBS supernatants of the various coatings yielded information about the release profiles of differently functionalized mesoporous silica films (Fig. 3). Released amounts of ciprofloxacin are given according to the macroscopic surface of the substrate. From the unmodified mesoporous silica layer loaded with ciprofloxacin, $0.2 \mu \mathrm{g} \mathrm{cm}^{-2}$ of the drug were released (Fig. 3, sample "A + CFX"). Upon modification with sulfonic acid groups, this value increased nearly ten-fold to $1.9 \mu \mathrm{g} \mathrm{cm}^{-2}$ (Fig. 3, sample C). The unmodified as well as the modified mesoporous layer both showed a typical initial burst release profile, where most of the drug was released within the first few hours, with just small amounts being released after the first $24 \mathrm{~h}$. After twelve days, practically all the ciprofloxacin, which can be released (after the maximum time of 63 days) from the sulfonate-modified layer, had been released. However, the release profiles could be tailored by further functionalization steps. Samples which were dip-coated with

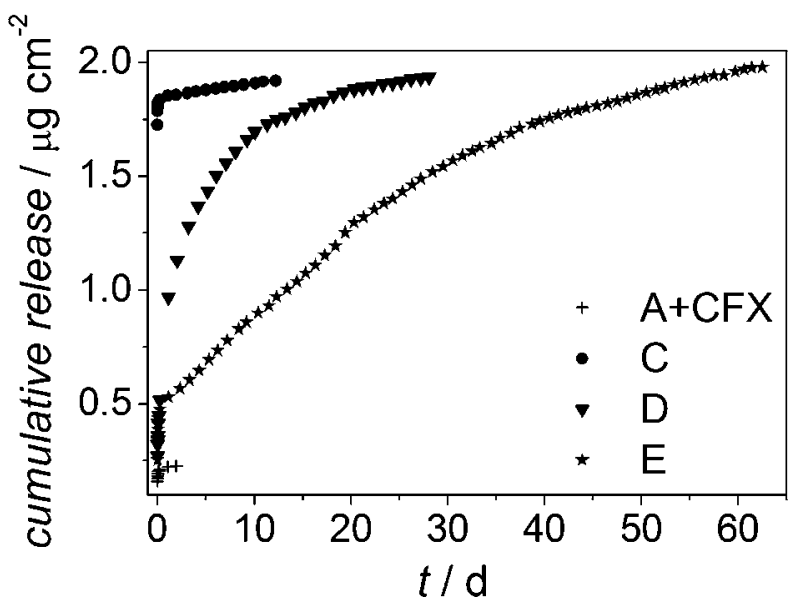

Fig. 3 Release profiles of ciprofloxacin-loaded mesoporous silica layers on glass substrates (A + CFX) functionalized successively with sulfonic acid groups (C), a dip-coated layer derived from bis(trimethoxysilyl)hexane (D) and a layer derived from dioctyltetramethyldisilazane (E) by evaporation coating. 
bis(trimethoxysilyl)hexane show a slower release (Fig. 3, sample D). After the first twelve days, approx. $90 \%$ of the total amount had been released. The release rate then decreases so that very small doses are still obtained up to 31 days. With the additional surface coating produced by the evaporation of dioctyltetramethyldisilazane on top of the sample, an even more prolonged release profile is established (Fig. 3, sample E). Here, after twelve days less than $50 \%$ of the total amount was released. After a small initial burst, a constant release rate was observed for more than 30 days, followed by regular smaller doses up to 63 days. The surface coatings did not influence the total amount of drug released, which in all cases was about $2 \mu \mathrm{g} \mathrm{cm} \mathrm{cm}^{-2}$ of ciprofloxacin. This fact demonstrates that only very small amounts of ciprofloxacin were lost during the additional functionalization steps. This could be a critical issue especially in the case of the dip-coating procedure where the drug-loaded sample is exposed to an aqueous solution, as it is in the following release experiments. A fast withdrawal speed of $c a .2 \mathrm{~cm} \mathrm{~s}^{-1}$ was used in this step to avoid leaching of the inserted ciprofloxacin.

The release profiles depicted in Fig. 3 all show different release rates in different time regions. During the initial burst, sample $\mathrm{C}$, which carried only the sulfonate modification, discharged approx. $95 \%$ of the total released amount, whereas the samples which were coated lost only approx. 30\%. The sample which was equipped with the dip-coated layer produced from bis(trimethoxysilyl)hexane only (sample D) showed a rather fast release in the first ten days and then a slower one afterwards. The behavior of sample E, which possesses an additional coating produced from evaporation coating by dioctyltetramethyldisilazane, is similar, but the release rates are lower and the change from medium to slow release occurs only after approx. 30 days.

\section{Biocompatibility testing}

For biocompatibility testing samples were investigated with a cell assay using the murine fibroblast cell line NIH3T3. Results are shown in Fig. 4 and 5. Samples were tested with (samples C, D, and E) and without ciprofloxacin loading (samples B, F, G, and H). Substrates coated with a mesoporous silica layer modified with sulfonic acid groups (sample B) and also loaded with ciprofloxacin (sample C) proved to be highly biocompatible in cell culture assays. Microscopic examination revealed that the cells could efficiently adhere and proliferate on the coating, even on the one releasing the loaded ciprofloxacin. Samples containing ciprofloxacin which were coated with an additional hydrophobic layer prepared from bis(trimethoxysilyl)hexane (sample D), and with an additional coating produced by evaporation of dioctyltetramethyldisilazane (sample E), exhibited a somewhat lesser degree of proliferation. Similarly, ciprofloxacin-free silica films coated with additional silane-derived layers showed reduced cell densities. Here the following samples were investigated: sample $\mathrm{F}$ with a layer prepared from bis(trimethoxysilyl)hexane, sample $G$ with two layers, derived from bis(trimethoxysilyl)hexane and subsequent dioctyltetramethyldisilazane evaporation, and sample $\mathrm{H}$, coated only using dioctyltetramethyldisilazane. Due to cracks appearing in these organosilane-derived coatings during the cell culture experiment and the hydrophobic nature of the surface, the cells

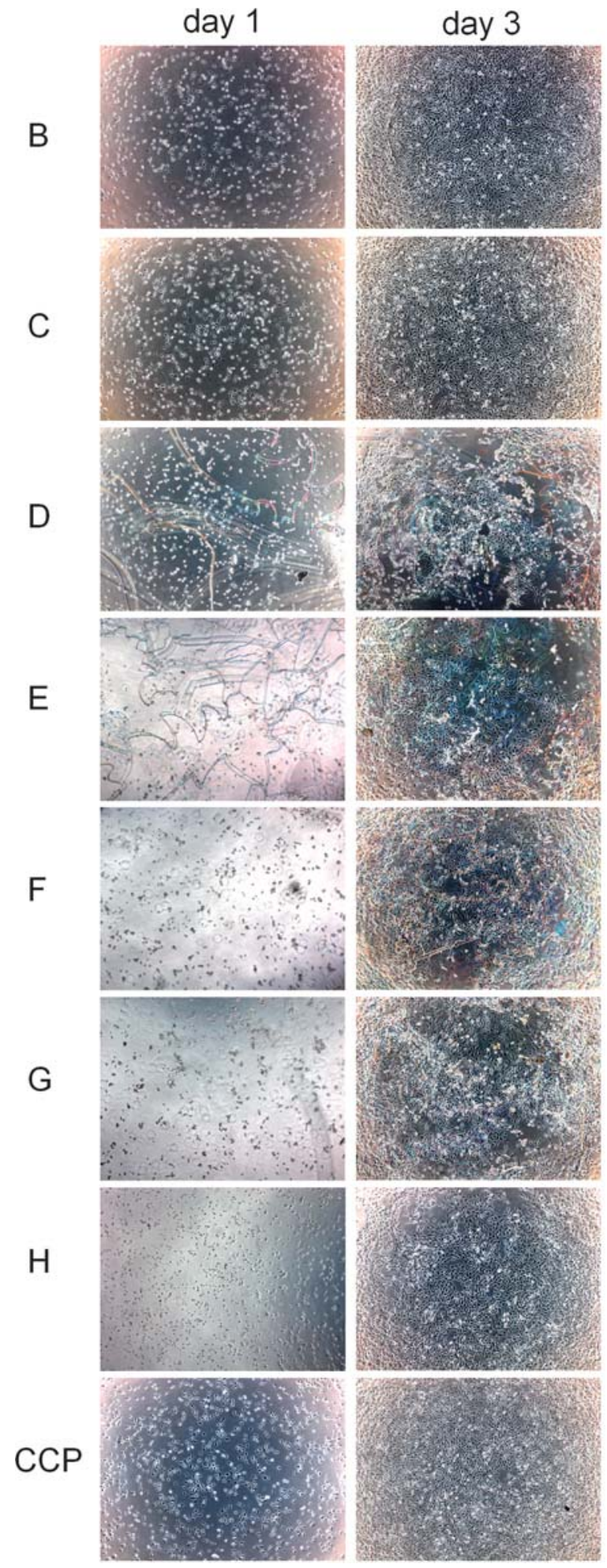

Fig. 4 Microphotographic images of cultured NIH3T3 fibroblast cells on modified mesoporous coatings taken after $24 \mathrm{~h}$ (day 1) and $72 \mathrm{~h}$ (day 3). Cell culture plastic (CCP) was used as a control; (B) sulfonated mesoporous silica films. Samples C, D and E were loaded with ciprofloxacin; samples F, G and $\mathrm{H}$ were used as ciprofloxacin-free controls. The films were further modified as follows: (D and F) dip-coated with bis(trimethoxysilyl)hexane; (E and G) dip-coated with bis(trimethoxysilyl)hexane and evaporation-coated with dioctyltetramethyldisilazane; (H) evaporation-coated with dioctyltetramethyldisilazane. 


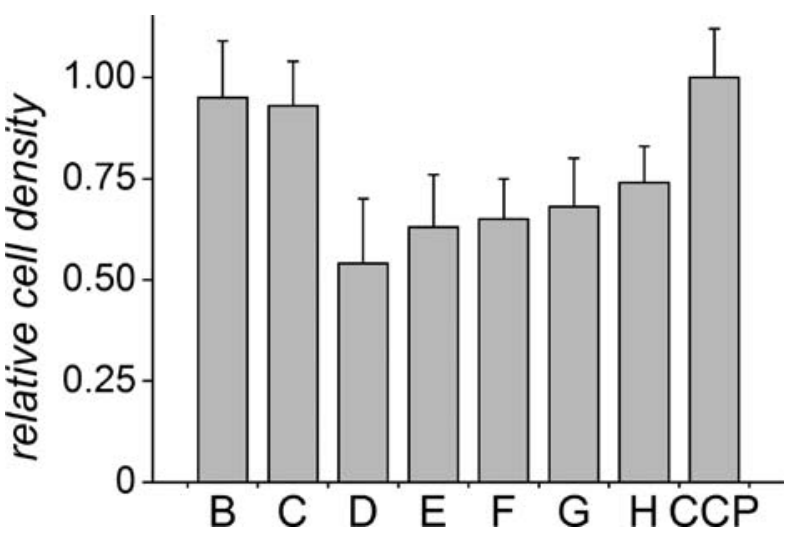

Fig. 5 Biocompatibility of ciprofloxacin-loaded mesoporous coatings inoculated with NIH3T3 fibroblast cells for $72 \mathrm{~h}$ (day 3) at $37^{\circ} \mathrm{C}$. The cell densities are given relative to the value of cells grown on cell culture plastic which was set to 1 (CCP). Samples C, D and E were loaded with ciprofloxacin; samples $\mathrm{F}, \mathrm{G}$ and $\mathrm{H}$ were used as ciprofloxacin-free controls. The films were further modified as follows: (D and F) dipcoated with bis(trimethoxysilyl)hexane; (E and $\mathrm{G}$ ) dip-coated with bis(trimethoxysilyl)hexane and evaporation-coated with dioctyltetramethyldisilazane; (H) evaporation-coated with dioctyltetramethyldisilazane.

were not able to adhere well on the surface after one day of incubation under cell culture conditions. After three days of incubation this effect was reduced. Thus, whereas all samples can be considered as biocompatible, this property is somewhat compromised in the case of the silane-derived surface coatings.

\section{Antibacterial efficacy}

The bacteria of the $P$. aeruginosa stem used (PAO1 CTX::lux) exhibit luminescence when alive. Results of experiments using these bacteria illustrate the antibacterial efficiency of ciprofloxacin-loaded samples. After six hours in LB medium, ciprofloxacin-loaded samples showed only about one-eighth of the bacterial luminescence in comparison to the values obtained with the mesoporous silica coating only, with the sulfonate-functionalized mesoporous silica and with a glass control (Fig. 6). These results show that the amount of ciprofloxacin of the delivery system is in principle appropriate to locally curb bacterial proliferation.

To test the efficacy of the time-dependent release from functionalized mesoporous silica films, we incubated disks of materials $\mathrm{C}, \mathrm{D}$ and $\mathrm{E}$ (see Fig. 1) in LB medium and collected the supernatants every $48 \mathrm{~h}$ up to ten days. The medium was replaced each time after removing the supernatant liquid. These samples collected were afterwards tested for their effect on the proliferation of the luminescent $P$. aeruginosa bacteria (i.e. in the absence of the drug delivery systems). In parallel, the release of ciprofloxacin was studied on an identical time-scale. For this purpose, corresponding samples were placed in PBS buffer solution, and the supernatant was collected and replaced every $48 \mathrm{~h}$ (due to the high absorbance of the LB medium, it was not possible to determine ciprofloxacin concentrations directly in this medium using our method). In this way, it was possible to correlate the amount of ciprofloxacin released and its effect on bacterial population in a simulated dynamic fluid environment (Fig. 7).

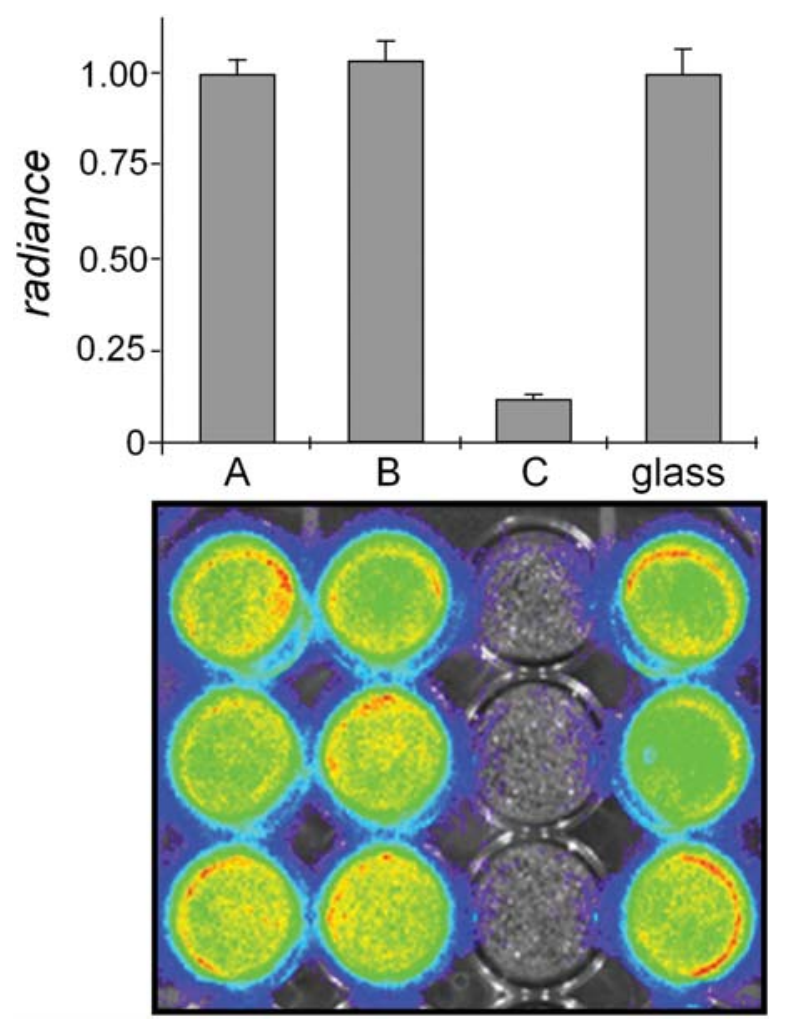

Fig. 6 Antibacterial efficacy of a ciprofloxacin-loaded mesoporous silica film against luminescent bacteria ( $P$. aeruginosa, PAO1 CTX::lux) after $6 \mathrm{~h}$ in vitro. (A) Mesoporous silica film on a glass substrate; (B) as A, but with sulfonic acid modification; (C) as B, but loaded with ciprofloxacin. A plain glass sample (glass) is used as a reference and the radiance is given relative to the value of this sample which was set to 1 .

The sulfonated mesoporous film loaded with ciprofloxacin (sample $\mathrm{C}$ in Fig. 7) released the antibiotic very fast in an initial burst. In the supernatant collected after two days, no proliferation of bacteria was observed whereas at later time points, the release of the residual minimal amounts of ciprofloxacin still delivered by the porous film cannot hinder the bacteria from an almost unrestricted proliferation of up to $90 \%$ of the control. The functionalized mesoporous silica film equipped with a slowrelease layer derived from bis(trimethoxysilyl)hexane (sample D in Fig. 7) shows no bacteria proliferation up to six days with a following slow increase of bacterial viability up to $40 \%$ of the control. This is consistent with the general release profile in vitro. The proliferation rate in the supernatants of the LB medium of silica coatings additionally functionalized by dioctyltetramethyldisilazane (sample E in Fig. 7) starts with $40 \%$ of the control after two days and decreases to approximately $10 \%$ after six days, followed by an increase up to $60 \%$ after ten days. This behavior indicates that the amount of ciprofloxacin released from these coatings is lower than that from the other samples, but these reduced amounts can still suffice to have an effect on bacterial growth. However, with this double silane-derived coating, the bacterial population does not decrease to practically zero at any point-of-time. The amounts of ciprofloxacin released in PBS show the same trend, but the largest dose is released here after eight days, somewhat later than in the LB medium 

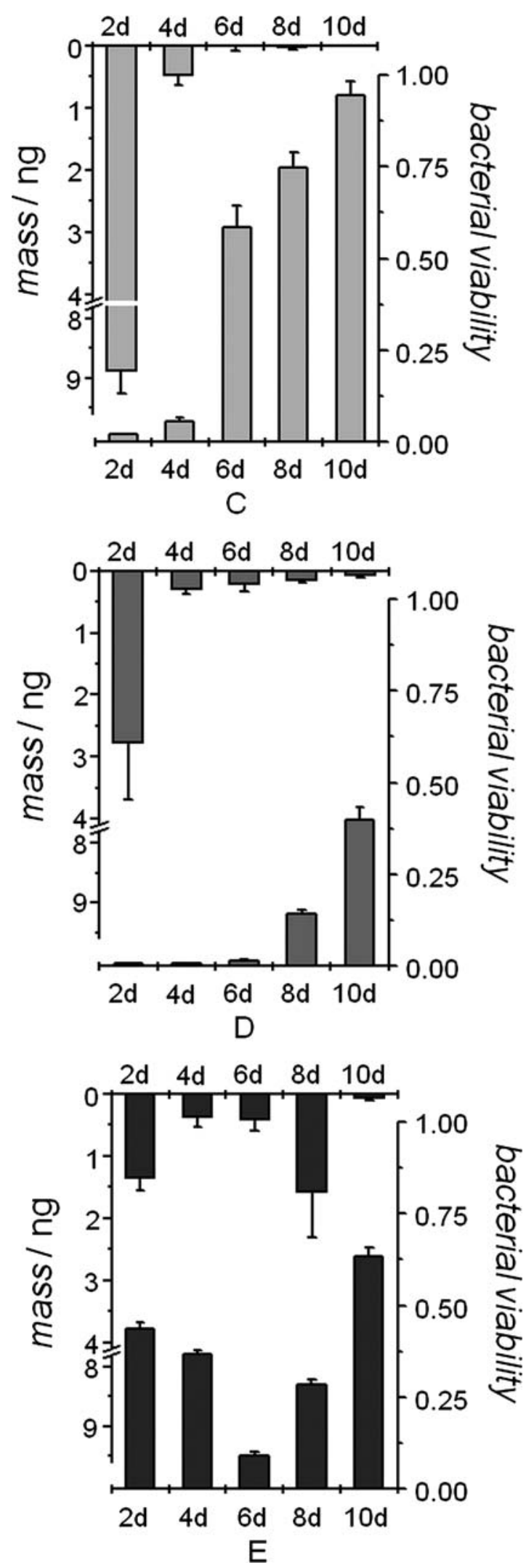

Fig. 7 Release profiles (top) and antibacterial efficacy (bottom) of ciprofloxacin loaded sulfonated mesoporous silica films. Sulfonated mesoporous silica films on glass substrates consecutively functionalized by loading with ciprofloxacin (C), by dip-coating with bis(trimethoxysilyl)hexane (D), and by evaporation coating with experiment. This reflects probably an effect of the different incubation media on the release kinetics. Furthermore, subtle differences in the handling or experimental procedures have to be considered.

These investigations show that it is possible to suppress bacterial proliferation for up to ten days by functionalized mesoporous silica films loaded with ciprofloxacin. The strong antibacterial activity of ciprofloxacin-loaded sulfonated mesoporous silica layers indicates that this material represents a promising practical approach to effectively fight bacterial infections of implants with very high local drug concentrations that would not be tolerable by systemic administration. The release of the antibiotic can be successfully controlled by the application of organosiloxane layers derived from bis(trimethoxysilyl)hexane which also possess a sufficient biocompatibility. ${ }^{30}$ According to our results, the additional application of a layer derived from dioctyltetramethyldisilazane appears to be less advisable, as the biocompatibility of such a coating layer is somewhat compromised and the amounts of ciprofloxacin released in a certain unit of time are quite low.

\section{Conclusions}

A general practical approach for the antibiotic defense directly from implants is presented. A sulfonated mesoporous silica film has a high loading capacity for the antibiotic ciprofloxacin. Additional layers of bis(trimethoxysilyl)hexane or dioctyltetramethyldisilazane can be applied to delay the release and to prolong the effective antibacterial activity period. Controlled release profiles can therefore be tailored using a fast dip-coating procedure, possibly in combination with an evaporation-coating approach. The general biocompatibility for all samples was demonstrated in cell culture experiments. Importantly, cells could even directly adhere to the ciprofloxacin-loaded sulfonated mesoporous silica films. The silane-derived surface coatings somewhat compromised the biocompatibility of the surface layers. Antibacterial efficacy was shown for a clinically relevant period of up to ten days, using pathogenic biofilm-forming bacteria. These results suggest that the development of drugloaded mesoporous silica films as coatings on medical implants is a promising approach to prevent bacterial implant infections during the most critical time span after implantation. The materials described here are currently being tested in mice and rabbit animal models, in the latter case with specific regard to middle-ear implants.

\section{Acknowledgements}

This work was supported by the DFG within the Collaborative Research Program SFB 599 "Sustainable bioresorbable and permanent implants based on metallic and ceramic materials". We thank our colleagues within work package D1 ("Functionalized Middle Ear Prostheses") for valuable discussions.

dioctyltetramethyldisilazane (E). Blank values determined on ciprofloxacin-free sulfonated mesoporous silica substrates were subtracted. Bacterial viability is given relative to the value obtained on sulfonated mesoporous silica films containing no drug, which was set to 1 (not shown). 
Furthermore we thank Dipl.-Chem. Sina Williams for support by developing drug release techniques.

\section{References}

1 B. Fink, Int. J. Med. Sci., 2009, 6, 287.

2 P. J. Sanderson, J. Hosp. Infect., 1991, 18(suppl. A), 367.

3 C. Turck, G. Brandes, I. Krueger, P. Behrens, T. Lenarz and M. Stieve, Acta Oto-Laryngol., 2007, 27, 801-808.

4 J. C. Vogt, G. Brandes, I. Krueger and P. Behrens, J. Mater. Sci.: Mater. Med., 2008, 19, 2629.

5 J. C. Vogt, G. Brandes, N. Ehlert, P. Behrens, I. Nolte, P. P. Müller, T. Lenarz and M. Stieve, J. Biomater. Appl., 2009, 24, 175-191.

6 J.-F. Chen, H.-M. Ding, J.-X. Wang and L. Shao, Biomaterials, 2004, 25, 723-727.

7 Y.-F. Zhu, J.-L. Shi, Y.-S. Li, H.-R. Chen, W.-H. Shen and X.P. Dong, Microporous Mesoporous Mater., 2005, 85, 75-81.

8 B. G. Trewyn, S. Giri, I. I. Slowing and V. S.-Y. Lin, Chem. Commun., 2007, 3236-3245.

9 G. Geyer, $H N O, 1999,47(2), 77-91$.

10 R. A. Goldenberg and J. R. Emmet, Otology \& Neurotology, 2001, 22, $45-152$.

11 P. Horcajada, A. Rámila, J. Pérez-Pariente and M. Vallet-Regí, Microporous Mesoporous Mater., 2004, 68, 105-109.

12 J. Andersson, J. Rosenholm, S. Areva and M. Lindén, Chem. Mater., 2004, 16, 4160-4167.

13 H. Böttcher, P. Slowik and W. Süß, J. Sol-Gel Sci. Technol., 1998, 13, 277-281.

14 M. Vallet-Regí, F. Balas and D. Arcos, Angew. Chem., Int. Ed., 2007, 46, 7548-7558.

15 W. Zeng, X.-F. Qian, J. Yin and Z.-K. Zhu, Mater. Chem. Phys., 2006, 97, 437-441.

16 S.-W. Song, K. Hidajat and S. Kawi, Langmuir, 2005, 21, 9568-9575.

17 B. Munoz, A. Rámila, J. Pérez-Pariente, I. Díaz and M. Vallet-Regí, Chem. Mater., 2003, 15, 500-503.

18 J. C. Doadrio, E. M. B. Sousa, I. Izquierdo-Barba, A. L. Doadrio, J. Perez-Pariente and M. Vallet-Regí, J. Mater. Chem., 2006, 16, $462-466$.
19 F. Qu, G. Zhu, S. Huang, S. Li and S. Qiu, ChemPhysChem, 2006, 7, 400-406.

20 Q. Tang, Y. Xu, D. Wu, Y. Sun, J. Wang, J. Xu and F. Deng, J. Controlled Release, 2006, 114, 41-46.

21 Q. Tang, Y. Xu, D. Wu and Y. Sun, J. Solid State Chem., 2006, 179, 1513-1520.

22 Q. Yang, S. Wang, P. Fan, L. Wang, Y. Di, K. Lin and F. S. Xiao, Chem. Mater., 2005, 17, 5999-6003.

23 F. Bals, M. Manzano, P. Horcajada and M. Vallet-Regí, J. Am. Chem. Soc., 2006, 128, 8116-8117.

24 C. Tourné-Péteilh, D. Brunel, S. Bégu, B. Chiche, F. Fajula, D. A. Lerner and J.-M. Devoiselle, New J. Chem., 2003, 27, 14151418.

25 P. Horcajada, A. Rámila, G. Férey and M. Vallet-Regí, Solid State Sci., 2006, 8, 1243-1249.

26 J. M. Rosenholm and M. Lindén, J. Controlled Release, 2008, 128, $157-164$.

27 M. Manzano, V. Aina, C. O. Areán, F. Balas, V. Cauda, M. Colilla, M. R. Delgado and M. Vallet-Regí, Chem. Eng. J., 2008, 137, 30-37.

28 A. Rámila, B. Munoz, J. Pérez-Pariente and M. Vallet-Regí, J. SolGel Sci. Technol., 2003, 26, 1199-1202.

29 P. Kortesu, M. Ahola, M. Kangas, T. Leino, S. Laakso, L. Vuorilehto, A. Yli-Urpo, J. Kiesvaara and M. Marvola, J. Controlled Release, 2001, 76, 227-238.

30 Z. Wu, Y. Jiang, T. Kim and K. Lee, J. Controlled Release, 2007, 119, 215-221.

31 P. N. De Aza, A. H. De Aza, P. Pena and S. De Aza, Bol. Soc. Esp. Ceram. Vidrio, 2007, 46, 46-55.

32 E. Beleites, G. Schneider, W. Fried, D. Schumann and W. Linß, Dtsch. Ärztebl., 2001, 98, A244-A248.

33 T. Yamada, H. Zhou, H. Uchida, I. Honma and T. Katsube, J. Phys. Chem. B, 2004, 108, 13341-13346.

34 R. Marschall, I. Bannat, J. Caro and M. Wark, Microporous Mesoporous Mater., 2007, 99, 190-196.

35 G. Betani, J. Bacteriol., 1951, 62, 293-300.

36 P. Ghosh, S. C. Chadha, A. R. Mukherjee and S. R. Palit, J. Polym. Sci., Part A: Gen. Pap., 1964, 2, 4433-4440.

37 N. Ehlert, P. P. Müller, M. Stieve and P. Behrens, Microporous Mesoporous Mater., 2010, 131, 51-57. 